\title{
Severe Vitamin D Deficiency Increases Mortality Among Patients With Liver Cirrhosis Regardless of the Presence of HCC
}

\author{
ANTONIO RICCARDO BUONOMO ${ }^{1}$, RICCARDO SCOTTO ${ }^{1}$, EMANUELA ZAPPULO ${ }^{1}$, \\ MARIAGIOVANNA NERILLI ${ }^{1}$, BIAGIO PINCHERA ${ }^{1}$, GIUSEPPE PERRUOLO ${ }^{2}$, \\ PIETRO FORMISANO ${ }^{2}$, SALVATORE NAPPA ${ }^{1}$ and IVAN GENTILE ${ }^{1}$ \\ ${ }^{1}$ Department of Clinical Medicine and Surgery, Section of Infectious Diseases, \\ Federico II University of Naples, Naples, Italy; \\ ${ }^{2}$ Department of Medical Translational Sciences, Federico II University of Naples, Naples, Italy
}

\begin{abstract}
Background: The aim of this study was to investigate the association between vitamin $D$ deficiency $(<10 \mathrm{mg} / \mathrm{ml})$ and mortality in patients with and without hepatocellular carcinoma (HCC) in a cohort of patients with liver cirrhosis. Materials and Methods: A prospective study was conducted among 345 patients with liver cirrhosis. Results: At enrolment, 46 (13.3\%) patients had HCC. Severe vitamin $D$ deficiency was associated with mortality $(p<0.01)$. At the survival analysis, alpha-fetoprotein $>10 \mathrm{ng} / \mathrm{ml}$ $(p=0.003)$, vitamin $D$ deficiency $(p<0.001)$, a Model for End-Stage Liver Disease score $\geq 15(p<0.001)$, Child-Pugh class $B$ and $C$ (versus $A)(p<0.001)$ and the presence of active HCC $(p<0.001)$ were strongly associated with death. At the multivariate Cox regression analysis, only Child-Pugh class $B$ and $C$ (versus $A$ ) and vitamin $D$ deficiency were found to be significantly associated with death during the follow-up period ( $p<0.001$ and $p=0.006$, respectively). Conclusion: Vitamin D deficiency is common in patients with $H C C$, it is associated with active HCC and it negatively affects the overall survival of patients with cirrhosis.
\end{abstract}

Hepatocellular carcinoma (HCC) is one of the major complications of liver cirrhosis. It is the sixth most-diagnosed

This article is freely accessible online.

Correspondence to: Antonio Riccardo Buonomo, MD, Department of Clinical Medicine and Surgery-Section of Infectious Diseases, University of Naples Federico II, Via S. Pansini 5, 80131 Naples, Italy. Mobile: +39 3282486870, e-mail: antonioriccardobuonomo@ gmail.com

Key Words: Cirrhosis, vitamin D deficiency, hepatocellular carcinoma, mortality, risk factors. malignancy worldwide (1) and its incidence among patients with liver cirrhosis is estimated to be $1-8 \%$ per year (2). Moreover, HCC is often diagnosed in an advanced stage and therefore treatment options are often limited (3). In patients with cirrhosis, several factors are associated with a higher risk of developing HCC, for instance, co-infection with hepatitis $\mathrm{C}$ virus (HCV)/human immunodeficiency virus (HIV)/hepatitis B virus (HBV), obesity, steatosis, diabetes mellitus, and alcohol abuse (4-7). The interest in new factors potentially involved in the occurrence of HCC increased recently due to evidence that some patients with chronic HCV infection may show a de novo occurrence of HCC during or after treatment with new directly acting antiviral agents, despite their high efficacy in curing viral infection (8-11). In fact, new anti-viral treatments for $\mathrm{HCV}$ are able to reach very high rates of viral eradication with scarce side-effects differently from previous antiviral treatments for HCV (interferon era) (12-14).

Vitamin D exerts several biological effects other than the wellknown regulation of calcium and phosphorus metabolism: it has a role in activating innate and adaptive immunity $(15,16)$ thus having a potential role in preventing infection, and it is also able to influence neo-angiogenesis $(17,18)$ and pathways of apoptosis $(19,20)$. Accumulating evidence has shown that vitamin D deficiency is associated with a higher risk of developing different types of cancer (21-23) and it may, thus, have a role in the diagnosis of HCC, together with other markers already known to be useful in the diagnosis of such neoplasia [e.g. alphafetoprotein (AFP), protein induced by vitamin $\mathrm{K}$ absence-II and their combination] (24). However, little is known about the relationship of vitamin D deficiency with HCC risk, and mortality among patients with liver cirrhosis. In a European nested-control study, low levels of serum vitamin D prediagnosis were associated with an increased risk of HCC development regardless of pre-existing liver disease (25), probably augmenting the mortality rate in such patients. 
The aim of our study was to investigate the association between vitamin $\mathrm{D}$ deficiency and mortality in patients with and without HCC in a cohort of patients with liver cirrhosis.

\section{Materials and Methods}

Study design. Inpatients and outpatients referring to the Department of Clinical Medicine and Surgery - Section of Infectious Diseases of the Federico II University Hospital of Naples were prospectively enrolled from May 1st 2013 to July 31st 2016. Inclusion criteria were: age $\geq 18$ years and a diagnosis of liver cirrhosis (regardless of etiology). Exclusion criteria were: i) oral supplementation with cholecalciferol (vitamin D) or derivates in the previous 12 months, ii) refusal of consent. Patients enrolled underwent follow-up visits every 6 months until April 30st 2017. Follow-up visits were performed before schedule whenever the patients required medical assistance, such as for the occurrence of a cirrhosis-related complication.

Diagnosis of cirrhosis was made by clinical criteria, histological findings, or a liver stiffness $\geq 13 \mathrm{kPa}$ at FibroScan ${ }^{\circledR}$ (26). Clinical criteria for the diagnosis of cirrhosis included both laboratory (hypoalbuminemia, thrombocytopenia, low pseudocholinesterase level, decrease in prothrombin activity) and ultrasonographic findings, as well as the occurrence of cirrhosis-related complications, namely jaundice, ascites and porto-systemic encephalopathy as reported elsewhere (27-29). Demographic and clinical data of all the enrolled patients were collected at baseline, together with blood samples for biochemistry and complete blood counts. One blood sample was collected from each patient for centralized determination of serum 25$\mathrm{OH}$-vitamin D. Vitamin D insufficiency was defined as serum 25-OHvitamin D concentrations between 20 and $30 \mathrm{ng} / \mathrm{ml}$, while deficiency and severe deficiency were defined as serum concentrations of $10-20 \mathrm{ng} / \mathrm{ml}$ and $\leq 10 \mathrm{ng} / \mathrm{ml}$, respectively (30).

Finally, the presence of HCC was investigated in each patient at enrollment. Patients with a diagnosis of $\mathrm{HCC}$ at enrollment were divided into those with active $\mathrm{HCC}$ and those with a previous diagnosis of HCC. Diagnosis of HCC was made by radiological, histological or cytological criteria, in accordance with the American Association for the Study of the Liver (AASLD) HCC guidelines (31). Among patients with active HCC at enrollment, both the presence of portal vein invasion and the presence of metastasis were investigated. At each follow-up visit, clinical information of the enrolled patients was collected. Moreover, all patients underwent ultrasonography every 6 months and, in cases of detection of a suspicious or typical lesion, they were managed according to AASLD guidelines (31) and the date of the diagnosis was recorded. Finally, last visit to our Department was evaluated to perform survival analysis. Missing data regarding the occurrence of HCC or serum 25$\mathrm{OH}$-vitamin D concentrations, as well as those lost to follow-up and those receiving vitamin D supplementation during the observational period, were considered exclusion criteria during the study.

Outcomes of the study and sample size. The primary outcome of the study was to estimate the incidence of HCC among patients with liver cirrhosis and severe deficiency of vitamin D.

Secondary outcomes were to: analyze the difference in $25-\mathrm{OH}-$ vitamin $\mathrm{D}$ concentrations between patients with and those without active HCC; analyze the risk factors for new-onset HCC among patients with liver cirrhosis; and estimate the mortality rate among patients with severe vitamin D deficiency and HCC.
Given an HCC incidence rate of $6 \%$ of among patients with liver cirrhosis, we estimated that to show an incidence rate at least 1.5 -fold higher among patients with severe vitamin D deficiency and liver cirrhosis the calculated sample size was 72 ( $\alpha$-error $=0.05$; power $=0.8$ ).

Statistical analysis. Kolmogorov-Smirnov test was applied to quantitative variables to check for Gaussian distribution. Data are given as mean \pm standard deviation or as median and interquartile range (IQR) in the case of Gaussian and non-Gaussian distribution, respectively. For comparisons of categorical variables, the chisquared test (or Fisher's exact test if appropriate) was used, while the $t$-test or the Mann-Whitney $U$-test were used for comparisons of continuous variables for Gaussian-distributed or non-Gaussiandistributed data, respectively. Logistic regression model was used to estimate the odds ratio (OR), while Poisson regression with robust variance estimator was used to estimate the incidence risk ratio (RR). The variables that were significantly associated at univariate analysis were included in the multivariate model analysis. The Kaplan-Meier model was used for survival analysis. The effect of single variables on survival was assessed using the log-rank test. The variables that showed a significant association in this test were included in the Cox regression multivariate test. For all tests, a twosided $p$-value of less than.05 was considered statistically significant. Statistical analysis was carried out using the Statistical Package for the Social Sciences version 2.0 (IBM, Armonk, NY, USA).

Ethical statement. The present prospective study was approved by the Ethical Committee of the Federico II University of Naples (Prot. No. 128/12) and was conducted in accordance with The Code of Ethics of the World Medical Association (Declaration of Helsinki, Sixth Version) for experiments involving humans. Written informed consent was obtained from each patient included in the study.

\section{Results}

Three-hundred and forty-five patients were enrolled, none of them was excluded during the study due to missing data on oral vitamin D supplementation during the study. Clinical features of enrolled patients are shown in Table I. At enrollment, 46/345 (13.3\%) patients had a diagnosis of HCC and, 23/46 of these (50\%) had active HCC, while the other half had a history of successfully treated HCC. Among patients with active HCC at enrollment, 9/23 (39.1\%) had local extension of the neoplasia to the portal vein, while $8 / 23$ $(34.8 \%)$ had extrahepatic metastasis. Table I also shows the clinical parameters of patients with and without active HCC. Patients with Child-Pugh class B or C cirrhosis showed an increased risk of HCC compared with those with class $\mathrm{A}$, as well as those with serum 25-OH-vitamin D concentrations $<10 \mathrm{ng} / \mathrm{ml}$. Moreover, the median serum vitamin D concentration was significantly lower among patients with HCC compared to those without. Finally, patients with a diagnosis of diabetes mellitus showed an increased risk of HCC, even though such a result was of borderline statistical significance $(\mathrm{OR}=2.18,95 \% \mathrm{CI}=0.91-5.27 ; p=0.082)$.

At baseline, the median serum 25-OH-vitamin D concentration was $15 \mathrm{ng} / \mathrm{ml}(\mathrm{IQR}=8-24 \mathrm{ng} / \mathrm{ml})$. Few patients 
Table I. Clinical and laboratory parameters of patients with and without active hepatocellular carcinoma (HCC).

\begin{tabular}{|c|c|c|c|c|c|}
\hline Parameter & All patients $(\mathrm{N}=345)$ & Active HCC $(n=23)$ & No HCC $(n=322)$ & OR $(95 \% \mathrm{CI})$ & $p$-Value \\
\hline Median age (IQR), years & $68(61-74)$ & $71(65-78)$ & $68(61-74)$ & $0.96(0.92-1.01)$ & 0.130 \\
\hline Male, n $(\%)$ & $268(77.7)$ & $17(73.9)$ & $184(57.1)$ & $2.12(0.82-5.53)$ & 0.743 \\
\hline \multicolumn{6}{|l|}{ Child-Pugh class, n (\%) } \\
\hline A & $268(77.7)$ & $10(43.5)$ & $258(8.1)$ & $0.19(0.08-0.45)$ & $<0.001$ \\
\hline $\mathrm{B}$ & $56(16.2)$ & $9(39.1)$ & $47(14.6)$ & $3.76(1.54-9.18)$ & 0.004 \\
\hline $\mathrm{C}$ & $21(6.1)$ & $4(17.4)$ & $17(5.3)$ & $3.78(1.16-12.34)$ & 0.028 \\
\hline \multicolumn{6}{|l|}{ Etiology of cirrhosis, $\mathrm{n}(\%)$} \\
\hline $\mathrm{HCV}$ & $335(97.1)$ & $23(100)$ & $312(96.9)$ & $\#$ & $\#$ \\
\hline $\mathrm{HBV}$ & $2(.6)$ & $0(0)$ & $2(.6)$ & \# & \# \\
\hline Alcohol abuse & $4(1.2)$ & $0(0)$ & $4(1.2)$ & \# & \# \\
\hline Cryptogenic & $4(1.2)$ & $0(0)$ & $4(1.2)$ & \# & \# \\
\hline Diabetes, n (\%) & $136(39.4)$ & $13(56.5)$ & $123(38.2)$ & $2.18(.91-5.27)$ & 0.082 \\
\hline Vitamin $\mathrm{D}<10 \mathrm{ng} / \mathrm{ml}, \mathrm{n}(\%)^{*}$ & $111(32.2)$ & $12(52.2)$ & $99(3.7)$ & $2.46(1.05-5.76)$ & 0.039 \\
\hline Median glucose (IQR), mg/dl & $101(88-120)$ & $101(89-135)$ & $101(87-120)$ & & 0.681 \\
\hline Median albumin (IQR), g/dl & $3.7(3.4-4.1)$ & $3.4(2.8-3.7)$ & $3.8(3.4-4.1)$ & & 0.146 \\
\hline Median creatinine (IQR), mg/dl & $1.0(1.0-1.0)$ & $1.5(1.0-1.5)$ & $1.0(1.0-1.0)$ & & 0.351 \\
\hline Median AST (IQR), IU/ml & $48(30-83)$ & $75(36-168)$ & $47(29-81)$ & & 0.013 \\
\hline Median ALT (IQR), IU/ml & $43(22-72)$ & $43(28-98)$ & $43(21-71)$ & & 0.438 \\
\hline Median total bilirubin (IQR), $\mathrm{mg} / \mathrm{dl}$ & $1.03(.7-1.6)$ & $1.4(.9-2.3)$ & $1.0(.7-1.5)$ & & 0.281 \\
\hline Median direct bilirubin (IQR), $\mathrm{mg} / \mathrm{dl}$ & $0.4(.2-.6)$ & $0.6(.3-1.8)$ & $0.4(.2-.6)$ & & 0.107 \\
\hline Median PCHE (IQR), mg/dl & $3,728(2,133-5,768)$ & $2,151(1,414-3,338)$ & $4,069(2,366-6,063)$ & & 0.160 \\
\hline Median WBCs (IQR), $\times 10^{3} / \mathrm{mm}^{3}$ & $5.115(3.852-6.622)$ & $5.200(1.230-8.510)$ & $5.110(3.845-6.500)$ & & 0.560 \\
\hline Median RBCs (IQR), $\times 10^{6} / \mathrm{mm}^{3}$ & $4.26(3.77-4.73)$ & $4.03(3.36-4.23)$ & $4.28(3.78-4.75)$ & & 0.016 \\
\hline Median $\mathrm{Hb}(\mathrm{IQR}), \mathrm{g} / \mathrm{dl}$ & $13.1(11.3-14.6)$ & $12.5(9.7-13.1)$ & $13.2(11.4-14.6)$ & & 0.002 \\
\hline Median PLTs (IQR), $\times 10^{3} / \mathrm{mm}^{3}$ & $109(74-156)$ & $102(62-156)$ & $109(75-156)$ & & 0.627 \\
\hline Median INR & $1.16(1.06-1.28)$ & $1.23(1.17-1.38)$ & $1.15(1.06-1.27)$ & & 0.195 \\
\hline Median AFP (IQR), ng/ml & $7.0(3.8-13.5)$ & $211.0(26.2-968.7)$ & $7.0(3-12.0)$ & & $<0.001$ \\
\hline Median vitamin D (IQR), ng/ml* & $15.0(8.0-24.0)$ & $9.0(4.9-17.0)$ & $15.0(8.0-24.0)$ & & 0.010 \\
\hline
\end{tabular}

OR: Odds ratio; CI: confidence interval; AST: aspartate-transaminases; ALT: alanine-transaminases; ALP: alkaline phosphatase; GGT: gamma-glutamyl transpeptidase; PCHE: pseudocholinesterase; WBCs: white blood cells; RBCs: red blood cells; Hb: hemoglobin; PLTs: platelets; INR: international normalized ratio; AFP: $\alpha$-fetoprotein. *25-OH-Vitamin D. "Not calculable, the etiology of cirrhosis was HCV for all patients with active HCC.

$(52 / 345,15 \%)$ were found to have a sufficient level of vitamin D (Figure 1).

During the follow-up (median=9 months, IQR=6-16 months), among the 299 patients without HCC at baseline, a total of 13 patients $(4.4 \%)$ had a diagnosis of de novo $\mathrm{HCC}$, while out of the whole cohort, 54 (15.7\%) died. Among patients with severe vitamin D deficiency, 6/111 (5.4\%) developed de novo HCC during the follow-up, while 7/234 (2.9\%) patients with serum 25-OH-vitamin D concentrations $>10 \mathrm{ng} / \mathrm{ml}$ had a new diagnosis of HCC $(p=0.162)$. The person-per-year incidence was 0.08 and 0.03 among patients with and without severe vitamin D deficiency, respectively. The RR of HCC among patients with severe vitamin D deficiency was 2.19 (95\% CI=0.71-6.73, $p=0.171)$.

AFP $>10 \mathrm{ng} / \mathrm{ml}$ was associated with HCC onset during the first 12 months of follow-up $(\mathrm{RR}=1.18 ; 95 \% \mathrm{CI}=1.16-89.91$, $p=0.036$ ) (Table II). In the survival analysis, AFP $>10 \mathrm{ng} / \mathrm{ml}$ $(p=0.003), 25-\mathrm{OH}$-vitamin $\mathrm{D}<10 \mathrm{ng} / \mathrm{ml}(p<0.001)$ (Figure $2)$, a Model for End-Stage Liver Disease score $\geq 15$ $(p<0.001)$, Child-Pugh class B and C (versus A) $(p<0.001)$ and the presence of active HCC $(p<0.001)$ were strongly associated with death.

At multivariate Cox regression analysis, which included all the covariates significantly associated with death by logrank test, only Child-Pugh class $\mathrm{B}$ and $\mathrm{C}$ (versus $\mathrm{A}$ ) and 25-OH-vitamin $\mathrm{D}<10 \mathrm{ng} / \mathrm{ml}$ were found to be significantly associated with death during the follow-up period $(\mathrm{HR}=6.27$; 95\% $\mathrm{CI}=2.09-18.77 ; p<0.001$ and $\mathrm{HR}=4.33$; $95 \% \mathrm{CI}=1.54-$ $12.20 ; p=0.006$, respectively) (Table III).

\section{Discussion}

In our cohort of patients with liver cirrhosis, we found a high prevalence $(64 \%)$ of vitamin D deficiency. This is probably due to the impairment of several mechanisms implicated in vitamin $\mathrm{D}$ absorption in advanced liver disease, such as activation and hydrolyzation of 25-OH-cholecalciferol or malabsorption due to portal hypertension. It is noteworthy that vitamin D deficiency is associated with a high rate of HCC at presentation. This finding can be explained by the known 


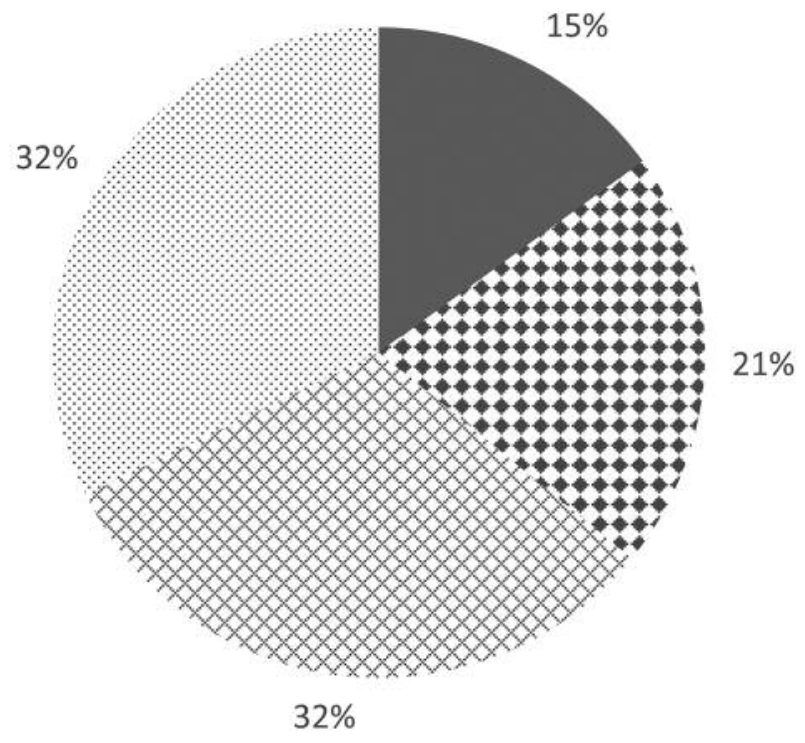

- Sufficiency ": Insufficiency

\section{× Deficiency Severe deficiency}

Figure 1. Rates of vitamin D insufficiency (25-OH-vitamin $D=20-30 \mathrm{ng} / \mathrm{ml}$ ), deficiency $(10-<20 \mathrm{ng} / \mathrm{ml})$ and severe deficiency $(<10 \mathrm{ng} / \mathrm{ml})$ in study patients $(N=345)$.

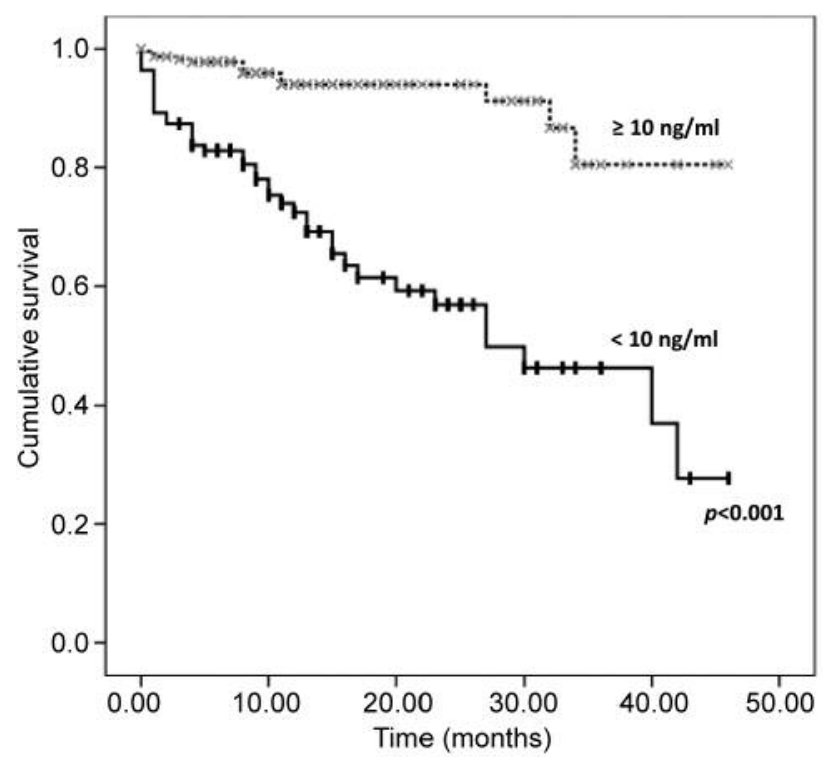

Figure 2. Cumulative survival in patients with and without severe deficiency of vitamin $D(25-\mathrm{OH}$-vitamin $\mathrm{D}<10 \mathrm{ng} / \mathrm{ml})$.
Table II. Univariate analysis of factors associated with hepatocellular carcinoma onset in the first 12 months of follow-up.

\begin{tabular}{lclc}
\hline Factor & RR & $95 \%$ CI & $p$-Value \\
\hline AFP $>10 \mathrm{ng} / \mathrm{ml}$ & 1.18 & $1.16-89.21$ & 0.036 \\
25-OH-Vitamin D <10 ng/ml & 2.19 & $0.71-6.73$ & 0.171 \\
Male sex & 1.19 & $0.38-3.72$ & 0.769 \\
MELD $>15$ & 3.41 & $0.87-13.37$ & 0.078 \\
Decompensated cirrhosis* & 1.49 & $0.39-5.65$ & 0.555 \\
Serum ferritin concentration & 1.00 & $0.99-1.00$ & 0.990 \\
\hline
\end{tabular}

RR: Incidence risk ratio; CI: confidence interval; AFP: $\alpha$-fetoprotein; MELD: Model for End-Stage Liver Disease score. *Child-Pugh class B or C.

Table III. Cox regression analysis for mortality.

\begin{tabular}{lllc}
\hline Factor & HR & \multicolumn{1}{c}{$95 \% \mathrm{CI}$} & $p$-Value \\
\hline AFP $>10 \mathrm{ng} / \mathrm{ml}$ & 2.03 & $0.82-5.07$ & 0.127 \\
$25-\mathrm{OH}-$ Vitamin D $<10 \mathrm{ng} / \mathrm{ml}$ & 4.33 & $1.54-12.20$ & 0.006 \\
MELD $\geq 15$ & 1.34 & $0.54-3.35$ & 0.524 \\
Child-Pugh B or C classification & 6.27 & $2.09-18.77$ & $<0.001$ \\
Active HCC & 2.23 & $0.88-5.66$ & 0.093 \\
\hline
\end{tabular}

HR: Hazard ratio; CI: confidence interval; AFP: $\alpha$-fetoprotein; MELD: model for end-stage liver disease score; HCC: hepatocellular carcinoma.

properties of vitamin $\mathrm{D}$, which has direct effects on cell differentiation and proliferation (32-34). We showed that HCC incidence was not significantly increased among patients with severe vitamin D deficiency. However, a significantly higher mortality was found in patients with liver cirrhosis and severe vitamin D deficiency compared with patients with serum 25$\mathrm{OH}$-vitamin D concentrations $>10 \mathrm{ng} / \mathrm{ml}$.

We underline that, even though it is known that vitamin D levels are lower in patients with advanced-stage liver disease according to Child-Pugh classification (35), in our cohort, severe deficiency of vitamin D remained associated with poor survival independently of Child-Pugh class (B or C versus $\mathrm{A}$ ) at multivariate analysis. However, this role might be totally independent of liver disease. In fact, vitamin D deficiency is found in many other diseases, for example in cardiovascular diseases; in this setting a large trial is currently ongoing (VITAL trial). This trial was designed to investigate the role of both vitamin D and omega-3 fatty acid supplementation in the reduction of heart diseases and stroke incidence in people without a prior history of these illnesses $(36,37)$. Results from these studies raise the hypothesis that the strong association between vitamin D deficiency and 
mortality is not only related to advanced liver disease. For instance, we showed that infection significantly reduces survival in patients with cirrhosis (38) and that vitamin D deficiency is associated with infection in such patients (35). Moreover, it is noteworthy that vitamin D deficiency affects the survival rate in patients with liver cirrhosis. In fact, there are different studies demonstrating this association but, to our knowledge, this is the first study demonstrating this association irrespective of the presence of $\mathrm{HCC}$, and in a sample of patients with low prevalence of post-alcoholic cirrhosis. Notably, in the abovementioned studies, the prevalence of patients with post-alcoholic liver cirrhosis was remarkably higher than in our study (from $36 \%$ to $61 \%$ in previous studies versus $1.2 \%$ in our study). Furthermore, it is conceivable that alcohol abuse may per se worsen prognosis of liver cirrhosis and affect vitamin D level.

Our study had certain limitations. Firstly, it was not powered enough to highlight possible correlation between severe vitamin D deficiency and active HCC. In fact, even though the prevalence of severe vitamin D deficiency was significantly higher among patients with active HCC, we cannot state that a cause-effect link is present. Moreover, as this was not an interventional study, we cannot state whether exogenous vitamin D supplementation may have a role in reducing mortality in patients with liver cirrhosis. In this regard, our study paves the way to further interventional research.

In conclusion, we demonstrated that in patients with liver cirrhosis, vitamin D deficiency was associated with poor survival irrespective of the presence of HCC. Therefore, randomized controlled trials aiming to confirm this association and demonstrate the potential role of vitamin D supplementation in improving survival rates are needed.

\section{Conflicts of Interests}

I.G. acted as a consultant for AbbVie and MSD and received a grant from Gilead Sciences (in the framework of Fellowship program). The other Authors have no conflicts of interest to declare. This research received no specific grant from any funding agency in the public, commercial, or not-for-profit sectors.

\section{References}

1 McGlynn KA and London WT: The global epidemiology of hepatocellular carcinoma: Present and future. Clin Liver Dis 15: 223-243, vii-x, 2011.

2 Ioannou GN, Splan MF, Weiss NS, McDonald GB, Beretta L and Lee SP: Incidence and predictors of hepatocellular carcinoma in patients with cirrhosis. Clin Gastroenterol Hepatol 5: 938-945, 2007

3 Sacco R, Gadaleta-Caldarola G, Galati G, Lombardi G, Mazza $\mathrm{G}$ and Cabibbo G: Easl hcc summit: Liver cancer management. Future Oncol 10: 1129-1132, 2014.

4 Dyal HK, Aguilar M, Bartos G, Holt EW, Bhuket T, Liu B, Cheung $\mathrm{R}$ and Wong RJ: Diabetes mellitus increases risk of hepatocellular carcinoma in chronic hepatitis $\mathrm{C}$ virus patients: A systematic review. Dig Dis Sci 61: 636-645, 2016.

5 Hagstrom H, Tynelius P and Rasmussen F: High BMI in late adolescence predicts future severe liver disease and hepatocellular carcinoma: A national, population-based cohort study in 1.2 million men. Gut 67: 1536-1542, 2017.

6 Younes R and Bugianesi E: Should we undertake surveillance for HCC in patients with NAFLD? J Hepatol 68: 326-334, 2017.

7 Suh B, Yun JM, Park S, Shin DW, Lee TH, Yang HK, Ahn E, Lee H, Park JH and Cho B: Prediction of future hepatocellular carcinoma incidence in moderate to heavy alcohol drinkers with the FIB-4 liver fibrosis index. Cancer 121: 3818-3825, 2015.

8 Gentile I, Maraolo AE, Buonomo AR, Zappulo E and Borgia G: The discovery of sofosbuvir: A revolution for therapy of chronic hepatitis C. Expert Opin Drug Discov 10: 1363-1377, 2015.

9 Lanini S, Scognamiglio P, Mecozzi A, Lombardozzi L, Vullo V, Angelico M, Gasbarrini A, Taliani G, Attili AF, Perno CF, De Santis A, Puro V, Cerqua F, D’Offizi G, Pellicelli A, Armignacco O, Mennini FS, Siciliano M, Girardi E, Panella V and Ippolito G: Impact of new DAA therapy on real clinical practice: A multicenter region-wide cohort study. BMC Infect Dis 18: 223, 2018.

$10 \mathrm{El}$ Kassas $\mathrm{M}$ and Funk AL: Increased recurrence rates of hepatocellular carcinoma after DAA therapy in a hepatitis Cinfected Egyptian cohort: A comparative analysis. J Viral Hepat 25: 625-630, 2017.

11 Buonomo AR, Gentile I and Borgia G: Direct acting antiviral agents and hepatocellular carcinoma development: Don't take it for granted. Transl Gastroenterol Hepatol 2: 101, 2017.

12 Tosone G, Borgia G, Gentile I, Cerini R, Conte MC, Orlando R and Piazza M: A case of pegylated interferon alpha-related diabetic ketoacidosis: Can this complication be avoided? Acta Diabetol 44: 167-169, 2007.

13 Gentile I, Viola C, Reynaud L, Borrelli F, Cerini R, Ciampi R, Piazza $\mathrm{M}$ and Borgia G: Hemolytic anemia during pegylated IFN-alpha2b plus ribavirin treatment for chronic hepatitis $c$ : Ribavirin is not always the culprit. J Interferon Cytokine Res 25: 283-285, 2005

14 Rosina F, Tosti ME, Borghesio E, Masocco M, Mele A, Coppola C, Milella M, Borgia G, Andreone P, Koch M, Zignego AL, Romano M, Carrara M, Almasio PL, Azzola E, Nardone G, Benedetti A, Carosi G, Mazzotta F, Sagnelli E and Rizzetto M: Pegylated interferon alpha plus ribavirin for the treatment of chronic hepatitis C: A multicentre independent study supported by the Italian Drug Agency. Dig Liver Dis 46: 826-832, 2014.

15 Bikle DD: Vitamin D and immune function: Understanding common pathways. Curr Osteoporos Rep 7: 58-63, 2009.

16 Wei R and Christakos S: Mechanisms underlying the regulation of innate and adaptive immunity by vitamin D. Nutrients 7: 8251-8260, 2015.

17 Flynn G, Chung I, Yu WD, Romano M, Modzelewski RA, Johnson CS and Trump DL: Calcitriol (1,25-dihydroxycholecalciferol) selectively inhibits proliferation of freshly isolated tumor-derived endothelial cells and induces apoptosis. Oncology 70: 447-457, 2006.

18 Chung I, Han G, Seshadri M, Gillard BM, Yu WD, Foster BA, Trump DL and Johnson CS: Role of vitamin D receptor in the antiproliferative effects of calcitriol in tumor-derived endothelial cells and tumor angiogenesis in vivo. Cancer Res 69: 967-975, 2009. 
$19 \mathrm{Ma}$ Y, Johnson CS and Trump DL: Mechanistic insights of vitamin d anticancer effects. Vitam Horm 100: 395-431, 2016.

20 Aggarwal A and Kallay E: Cross talk between the calciumsensing receptor and the vitamin D system in prevention of cancer. Front Physiol 7: 451, 2016.

21 Horas K, Maier G, Jakob F, Maus U, Kurth A, Jakuscheit A, Rudert $\mathrm{M}$ and Holzapfel BM: High prevalence of vitamin D deficiency in patients with bone tumors. Cancer Invest: 1-7, 2017.

22 Welsh J: Vitamin D and breast cancer: Past and present. J Steroid Biochem Mol Biol 177: 15-20, 2017.

23 Sy AM and Bautista JE: Association between serum vitamin D levels and colonic carcinomatous polyps. J Gastrointest Cancer 44: 481-485, 2013

24 Gentile I, Buonomo AR, Scotto R, Zappulo E, Carriero C, Piccirillo M, Izzo F, Rizzo M, Cerasuolo D, Borgia G and Cavalcanti E: Diagnostic accuracy of PIVKA-II, alphafetoprotein and a combination of both in diagnosis of hepatocellular carcinoma in patients affected by chronic HCV infection. In Vivo 31: 695-700, 2017.

25 Fedirko V, Duarte-Salles T, Bamia C, Trichopoulou A, Aleksandrova K, Trichopoulos D, Trepo E, Tjonneland A, Olsen A, Overvad K, Boutron-Ruault MC, Clavel-Chapelon F, Kvaskoff M, Kuhn T, Lukanova A, Boeing H, Buijsse B, Klinaki E, Tsimakidi C, Naccarati A, Tagliabue G, Panico S, Tumino R, Palli D, Bueno-de-Mesquita HB, Siersema PD, Peters PH, Lund E, Brustad M, Olsen KS, Weiderpass E, Zamora-Ros R, Sanchez MJ, Ardanaz E, Amiano P, Navarro C, Quiros JR, Werner M, Sund M, Lindkvist B, Malm J, Travis RC, Khaw KT, Stepien M, Scalbert A, Romieu I, Lagiou P, Riboli E and Jenab M: Prediagnostic circulating vitamin D levels and risk of hepatocellular carcinoma in European populations: A nested case-control study. Hepatology 60: 1222-1230, 2014.

26 Castera L, Forns X and Alberti A: Non-invasive evaluation of liver fibrosis using transient elastography. J Hepatol 48: 835-847, 2008.

27 Gentile I, Buonomo AR, Zappulo E and Borgia G: Is it possible to predict $\mathrm{HCV}$-related liver cirrhosis non-invasively through routine laboratory parameters? Infez Med 22: 11-18, 2014.

28 Gentile I, Coppola N, Pasquale G, Liuzzi R, D’Armiento M, Di Lorenzo ME, Capoluongo N, Buonomo AR, Sagnelli E, Morisco $\mathrm{F}$, Caporaso $\mathrm{N}$ and Borgia $\mathrm{G}$ : A simple noninvasive score based on routine parameters can predict liver cirrhosis in patients with chronic hepatitis C. Hepatitis monthly 13: e8352-e8352, 2013.
29 Lok AS, Ghany MG, Goodman ZD, Wright EC, Everson GT, Sterling RK, Everhart JE, Lindsay KL, Bonkovsky HL, Di Bisceglie AM, Lee WM, Morgan TR, Dienstag JL and Morishima C: Predicting cirrhosis in patients with hepatitis C based on standard laboratory tests: Results of the HALT-C cohort. Hepatology 42: 282-292, 2005.

30 Holick MF: Vitamin D status: Measurement, interpretation, and clinical application. Ann Epidemiol 19: 73-78, 2009.

31 Bruix J and Sherman M: Management of hepatocellular carcinoma: An update. Hepatology 53: 1020-1022, 2011.

32 Deeb KK, Trump DL and Johnson CS: Vitamin D signalling pathways in cancer: Potential for anticancer therapeutics. Nat Rev Cancer 7: 684-700, 2007.

33 Lamprecht SA and Lipkin M: Chemoprevention of colon cancer by calcium, vitamin D and folate: Molecular mechanisms. Nat Rev Cancer 3: 601-614, 2003.

34 Fedirko V, Bostick RM, Flanders WD, Long Q, Sidelnikov E, Shaukat A, Daniel CR, Rutherford RE and Woodard JJ: Effects of vitamin $\mathrm{D}$ and calcium on proliferation and differentiation in normal colon mucosa: A randomized clinical trial. Cancer Epidemiol Biomarkers Prev 18: 2933-2941, 2009.

35 Buonomo AR, Zappulo E, Scotto R, Pinchera B, Perruolo G, Formisano P, Borgia G and Gentile I: Vitamin D deficiency is a risk factor for infections in patients affected by HCV-related liver cirrhosis. Int J Infect Dis 63: 23-29, 2017.

36 Pradhan AD and Manson JE: Update on the vitamin D and omega3 trial (VITAL). J Steroid Biochem Mol Biol 155: 252-256, 2016.

37 Okereke OI and Manson JAE: Rationale and design of a largescale randomized controlled trial. Chapter 105-the vitamin D and omega-3 trial (VITAL): In: Vitamin D (third edition), pp. 20432055, 2011

38 Gentile I, Buonomo AR, Scotto R, Zappulo E and Borgia G: Infections worsen prognosis of patients with cirrhosis irrespective of the liver disease stage. Eur J Intern Med 46: e45-e47, 2017.

Received September 10, 2018

Revised October 8, 2018

Accepted October 12, 2018 